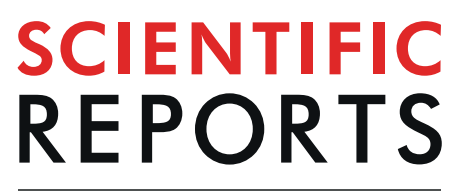

natureresearch

Check for updates

\title{
OPEN Alterations in Salience Network Functional Connectivity in Individuals with Restless Legs Syndrome
}

\begin{abstract}
Jeonghun $\mathrm{Ku}^{1}$, Yeong Seon Lee ${ }^{2}$, Keun Tae Kim², HyukWon Chang ${ }^{3} \&$ Yong Won Cho ${ }^{2 凶}$
Background and purpose: Restless legs syndrome (RLS) is a neurological disorder which is most commonly identified by an urge to move the legs. It often shows alterations in sensory processing which implies the salience network (SN) is experiencing changes. This study investigates the functional connectivity (FC) between the $S N$ and other areas of the brain in RLS patients during the resting state period. Methods: Thirty patients with drug naïve idiopathic RLS and 30 healthy age and gender matched controls were included in this study. Resting state fMRIs were performed in the morning during the asymptomatic period. The SN comparisons were conducted between the two groups. Results: The RLS group showed a reduction in SN FC in the right pyramis, and an increase in SN FC in the bilateral orbitofrontal gyri and right postcentral gyrus. Conclusions: The results of this study give reason to believe that SN FC in RLS patients is altered during asymptomatic periods. This could have an influence on the processing of the saliency of information, particularly sensory information processing and inhibition mechanisms.
\end{abstract}

Restless legs syndrome (RLS) is a neurological disorder in which patients often report an urge to move the legs (i.e., akathisia) ${ }^{1}$. The prevalence of RLS is $3.9-11.5 \%$ in the general population ${ }^{2,3}$. Two major putative causes for RLS are dopaminergic abnormality and iron insufficiency in the brain. Pharmacological therapy commonly includes drugs, such as dopamine agonists, iron, benzodiazepines, opiates, and alpha-2-delta ligands ${ }^{4}$. Leg akathisia is typically triggered by rest or a reduction in arousal ${ }^{1}$. It leads to alterations in the sensory processing mechanism of peripheral and brain responses. Brain imaging studies showed that there are morphologic changes in the primary somatosensory system ${ }^{5}$. Additionally, fMRI studies conducted on the thalamic and default mode networks have shown clear differences when compared to healthy subjects ${ }^{6,7}$, with varying results throughout the day $^{8}$. In addition, other functional connectivity studies suggest that RLS is associated with impaired attentional control of sensory input ${ }^{9}$. A neurophysiological study showed that RLS patients have a deficit of inhibitory modulation of the primary motor cortex ${ }^{10}$. Those studies consistently suggest that RLS can alter sensory information processing and sensory motor integration, and can activate the brain's compensation mechanism to inhibit sensations. These alterations may lead to abnormal sensory information processing, so RLS patients may experience unusual sensations and show lower current perception threshold (CPT) values. The studies also suggest impaired central sensory processing in RLS patients ${ }^{11}$. In addition, electrophysiological evidence suggests that sensory processing deficits are particularly related to the excitability of the early somatosensory gating control and an attenuated inhibitory interneuron network ${ }^{12}$. Thus, RLS patients may be more sensitive in perceiving sensations than the average person.

All these sensory processing deficits can be influenced by alterations in the patient's perception of saliency. In RLS patients, non-salient events seem to be coded in the brain as salient, so the patient cannot properly identify salient stimuli as opposed to non-salient stimuli ${ }^{13}$. This may result in the subject having a lower threshold for perceiving sensory stimuli ${ }^{11}$.

\footnotetext{
${ }^{1}$ Department of Biomedical Engineering, Keimyung University School of Medicine, Dongsan Medical Center, Daegu, South Korea. ${ }^{2}$ Department of Neurology, Keimyung University School of Medicine, Dongsan Medical Center, Daegu, South Korea. ${ }^{3}$ Department of Radiology, Keimyung University School of Medicine, Dongsan Medical Center, Daegu, South Korea. ${ }^{\bowtie e}$-mail: neurocho@gmail.com
} 
The Salience Network (SN) is the part of the brain that detects and filters salient stimuli and recruits relevant functional networks ${ }^{14}$. It plays a role in detecting and integrating emotional and sensory stimuli, while simultaneously switching between the internally directed cognition of the default mode network and the externally directed cognition of the central executive network (CEN).

Using this perspective of the role of the $\mathrm{SN}$, it could be assumed that the $\mathrm{SN}$ influences the other brain networks including the processing of sensory information in RLS patients. Perceptions traveling through the SN may possibly be altered and the connectivity of brain regions with the $\mathrm{SN}$ may also be altered, which would allow RLS symptoms to develop as the network attempts to process excessively non-salient stimuli. The purpose of this study was to investigate the alterations of the SN FC in RLS patients during the resting state to obtain further insight into the pathophysiology of RLS.

\section{Methods}

Study population. This study recruited 30 idiopathic RLS patients who had never received drug treatment for their condition (i.e, drug naïve) who visited a tertiary hospital outpatient sleep disorder center. It also included 30 age and gender matched healthy control subjects who had not experienced any previous sleep problems or other medical disorders. The control subjects all answered "no" on the initial questions of the RLS Diagnostic Questionnaire ${ }^{3,15}$. All patients were individually diagnosed by a certified Korean neurologist and RLS expert (YWC) through face-to-face interviews which utilized the Korean-language version ${ }^{3}$ of the Johns Hopkins Telephone diagnostic questionnaire ${ }^{15}$. All mimic diseases were excluded, as recommended by the updated international restless legs syndrome study group (IRLSSG) diagnostic criteria ${ }^{16}$. Patients diagnosed with secondary RLS because of iron-deficiency anemia, pregnancy, chronic kidney disease, peripheral neuropathy, or myelopathy were excluded. However, there were some cases of patients who only had peripheral iron deficiency with no definite cause. These patients were included in the study and their condition was not controlled for as with other RLS studies. All patients were untreated prior to enrolling in this study. The severity of RLS symptoms were evaluated using the validated Korean-language version ${ }^{17}$ of the International RLS scale (K-IRLS) ${ }^{18}$. Patients with circadian sleep disorders, parasomnia, sleep apnea, or other comorbid sleep disorders were identified using validated Korean-language versions of sleep questionnaires, including the Insomnia Severity Index (ISI-K) ${ }^{19}$, the Pittsburgh Sleep Quality Index (PSQI-K) ${ }^{20}$, and the Epworth Sleepiness Scale (ESS-K) ${ }^{21}$. Polysomnography was performed if needed, and all patients who reported comorbid sleep disorders were excluded.

The study was approved by the institutional review board of the Keimyung University Dongsan Hospital. Informed consent was obtained from all participating subjects. All methods were carried out in accordance with relevant guidelines and regulations.

MRI protocol. Resting state fMRI scans were conducted in the morning between 09:00-12:00, in order to minimize influence from RLS symptoms. A 3 T MRI Signa Excite scanner (GE Healthcare, Milwaukee, WI, USA) with an 8-channel high-resolution brain coil was used for this study. Each subject underwent an anatomic image series scan using a three-dimensional spoiled gradient-echo sequence (repetition time $(\mathrm{TR})=6 \mathrm{~ms}$, echo time $(\mathrm{TE})=2.2 \mathrm{~ms}$, flip angle $=20^{\circ}$, field of view $=240 \mathrm{~mm}, 256 \times 256,152$ axial slices, slice thickness $=2 \mathrm{~mm}$ thick). The functional images were scanned using a gradient echo planar imaging (EPI) sequence (TR $=2000$ $\mathrm{ms}, \mathrm{TE}=17.6 \mathrm{~ms}$, flip angle $=90^{\circ}$, field of view $=240 \mathrm{~mm}$, matrix $=64 \times 64$, slice thickness $=4 \mathrm{~mm}$, no gap, 244 scans of 30 contiguous axial slices) for approximately 8 minutes. Subjects were instructed to close their eyes without sleeping while maintaining a resting state for the duration of the scan. Patients who were unable to do so would be excluded, although there were no such instances during this study. In addition, none of the patients in this study reported RLS symptoms immediately before, during, or immediately after the MRI was administered.

Analysis of resting-state fMRI. Resting-state fMRI data was analyzed using the Analysis of Functional Neuroimage (AFNI) software ${ }^{22}$. The time series dataset discarded the first four time points, as is standard. The analysis included slice time correction for interleaved acquisitions, despiking, three-dimensional motion correction (allowing for head movement of $<2.5 \mathrm{~mm}$ ), temporal normalizing, linear and quadratic detrending, spatial normalization using the Montreal Neurological Institute (MNI) 152 template provided in the AFNI package, spatial smoothing (full width at half maximum $6 \mathrm{~mm}$ ), and temporal filtering $(0.009-0.1 \mathrm{~Hz})$.

The target region was defined as a $5 \mathrm{~mm}$ seed sphere in the right anterior insula (Fig. 1) because the right anterior insula has been previously identified as a crucial node of the $\mathrm{SN}^{23}$. Because there is no standard seed size, and typical variation is between $4 \mathrm{~mm}^{24}$ and $6 \mathrm{~mm}^{25}$, the seed was set as $5 \mathrm{~mm}$. Whole-brain voxel-wise correlations associated with the mean time series for the right insula were derived for each participant using $3 \mathrm{dDecon}$ volve (AFNI). For this step, the software identified and regressed 27 separate predictor modeling nuisance signals (white matter, cerebrospinal fluid, global signal, and 24 motion parameters ${ }^{26-28}$ ). Using the motion parameters, framewise displacement (FD $)^{29}$ was calculated and the average value of the FD was used as covariates in the group analysis step. The FD values were between 0.0412 and 0.2570 so nobody was excluded due to head movement. Each participant's correlation maps were converted to $\mathrm{z}$ score maps (Fisher r-to-z transformation) representing the strength of FC between brain areas and the seed region, which were then used to analyze group data.

An analysis of covariance (adjusted for FD, HAS and HDS) was used to evaluate differences in SN FC in the control group as compared to RLS patients. The $3 \mathrm{dClustSim}$ command provided in the AFNI analysis program was used to get the corrected $\mathrm{p}$ value of 0.05 with the threshold value of the cluster size being 800 microliters (100 voxels) under the uncorrected $p$ value of 0.01 . The strength of the connectivity from each brain region was compared with the patients' characteristics, which included symptom duration, age of onset, K-IRLS total, and sub-scores of K-IRLS ${ }^{30}$ (significance for these exploratory analyses was set at $p<0.05$ ). 


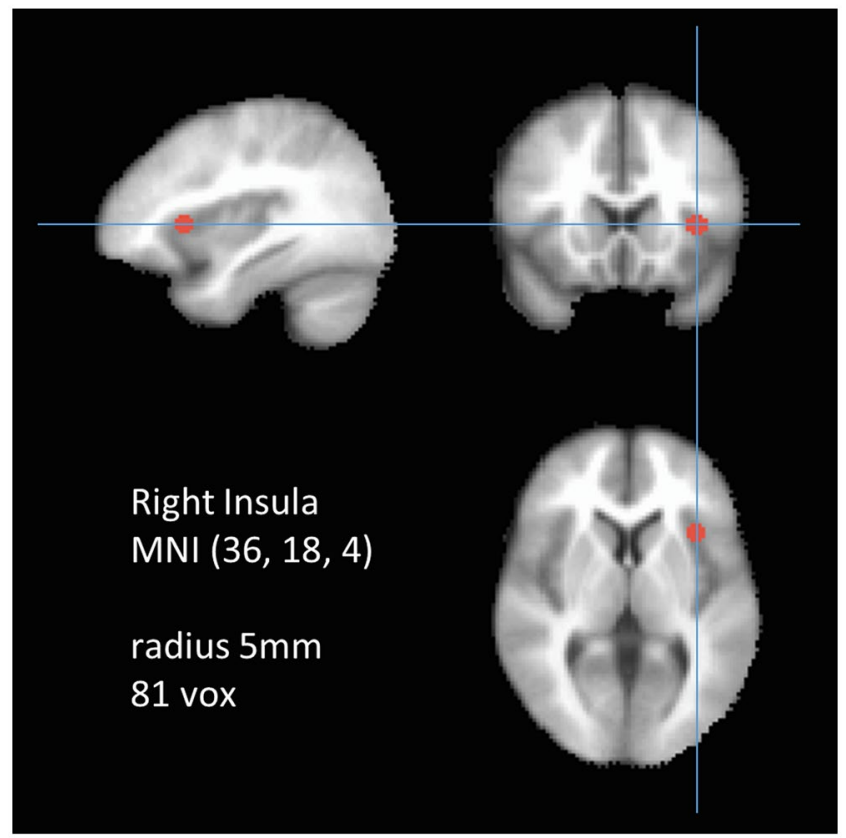

Figure 1. The seed region of interest was marked as $5 \mathrm{~mm}$ radius in the right insula at $(36,18,4)$ in MNI coordinates.

\begin{tabular}{|l|l|l|l|l|}
\hline & $\begin{array}{l}\text { Drug naïve RLS patients } \\
(\mathrm{n}=30)\end{array}$ & Controls $(\mathrm{n}=30)$ & $\chi^{2} / t$ & $p$-value \\
\hline Females (\%) / males (\%) & $23(76.7) / 7(23.3)$ & $19(63.3) / 11(36.7)$ & 1.270 & 0.260 \\
\hline Age (years) & $53.8 \pm 10.6$ & $53.4 \pm 11.2$ & 0.142 & 0.887 \\
\hline RLS severity (K-IRLS) & $26.4 \pm 6.9$ & & & \\
\hline symptom severity & $19.3 \pm 4.8$ & & & \\
\hline disease-specific QoL & $9.3 \pm 3.1$ & & & \\
\hline Age of onset & $43.2 \pm 15.0$ & & & \\
\hline Symptom duration (month) & $133.4 \pm 134.9$ & & & \\
\hline PSQI-K & $11.1 \pm 4.0$ & $4.0 \pm 1.4$ & 9.143 & $<0.001$ \\
\hline ISI-K & $16.2 \pm 6.7$ & $3.5 \pm 2.5$ & 9.792 & $<0.001$ \\
\hline ESS-K & $6.3 \pm 4.6$ & $4.5 \pm 3.3$ & 1.51 & 0.138 \\
\hline HAS & $7.7 \pm 4.3$ & $3.5 \pm 1.9$ & 4.919 & $<0.001$ \\
\hline HDS & $8.3 \pm 3.7$ & $5.1 \pm 2.7$ & 3.863 & $<0.001$ \\
\hline
\end{tabular}

Table 1. Demographic and clinical characteristics. The values are mean \pm SD. RLS: restless legs syndrome, K-IRLS: Korean version of the international RLS severity scale, QoL: quality of life, PSQI-K: Korean version of the Pittsburgh sleep quality index, ISI-K: Korean version of insomnia severity index, ESS-K: Korean version of Epworth sleepiness scale, HAS: Hospital Anxiety Scale, HDS: Hospital Depression Scale.

\section{Results}

The mean age of the RLS patients and controls was $53.8 \pm 10.6$ and $53.4 \pm 11.2$, respectively. There were 23 RLS females $(76.7 \%)$ and 19 control females $(63.3 \%)$. The RLS and control groups did not differ significantly in age or gender (Table 1). The mean ( \pm sd) K-IRLS was $26.4 \pm 6.9$, and the mean ( \pm sd) RLS duration was $133.4 \pm 134.9$ months. A summary of the sleep assessments for the RLS control groups is included in Table 1 (Figs. S1-9). RLS patients and controls showed no abnormalities, nor any brain atrophy on their T1, T2, or diffusion images, as confirmed by a neuro-radiologist.

During the asymptomatic period, the RLS group showed a reduction in SN connectivity in the right pyramis (Group mean and std. values across subjects of FC values across all voxels in the cluster were $-0.1617 \pm 0.1232$ for RLS and $-0.0159 \pm 0.1466$ for controls, which is placed at $(40,-68,-42)$ in the MNI coordinate with 1040 cluster size showing Max t value of -3.5817). There was an increase in SN connectivity for the bilateral orbitofrontal gyri (Group mean and std. values across subjects of FC values across all voxels in the cluster were $0.1527 \pm 0.1174$ for RLS and $0.0140 \pm 0.0988$ for controls, which is placed at $(-14,24,-18)$ in the MNI coordinate with 2232 cluster size showing Max t value of 3.8768 for the left; Group mean and std. values across subjects of FC values across all voxels in the cluster were $0.1197 \pm 0.1544$ for RLS and $-0.0403 \pm 0.1855$ for controls at $(6,18,-14)$ in the MNI coordinate with 856 cluster size showing Max $t$ value of 3.6056 for the right) and right postcentral gyrus 


\begin{tabular}{|l|l|l|l|l|l|}
\hline Anatomical region & \#Volume & X & Y & Z & Max $t$ \\
\hline lower connectivity in RLS & 1040 & 40 & -68 & -42 & -3.5817 \\
\hline Right Pyramis & 2232 & -14 & 24 & -18 & 3.8768 \\
\hline higher connectivity in RLS & 1400 & 62 & -32 & 44 & 3.7687 \\
\hline $\begin{array}{l}\text { Left Orbitofrontal } \\
\text { Gyrus }\end{array}$ & 856 & 6 & 18 & -14 & 3.6056 \\
\hline $\begin{array}{l}\text { Right Postcentral } \\
\text { Gyrus }\end{array}$ & $\begin{array}{l}\text { Right Orbitofrontal } \\
\text { Gyrus }\end{array}$ & &
\end{tabular}

Table 2. Patterns of connectivity in restless legs syndrome.

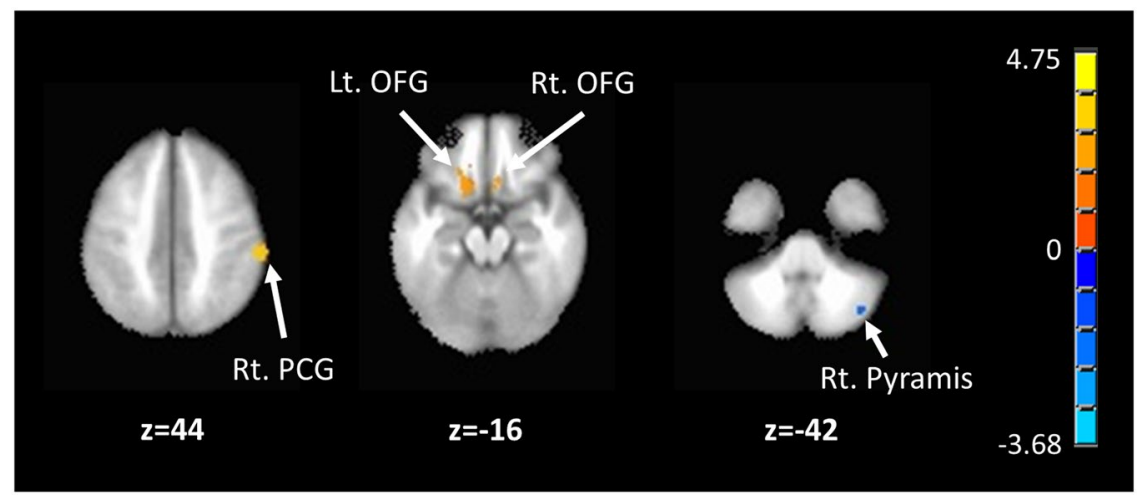

Figure 2. The areas showed differences the connectivity of the right anterior insula between the RLS group and healthy controls. The blue color represents the reduced connectivity of the $\mathrm{SN}$, while the red areas indicate increased connectivity of the SN in the RLS patients. Lt., left; Rt., right; OFG, orbitofrontal gyrus; PCG, postcentral gyrus.

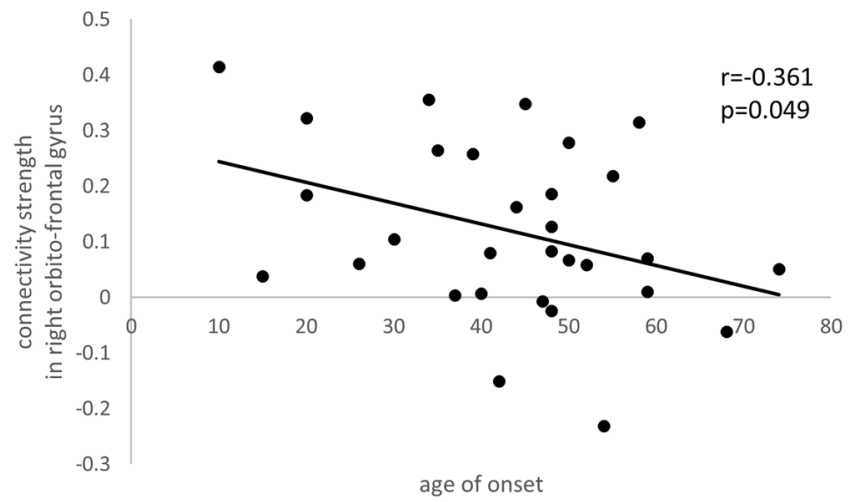

Figure 3. The correlation between the connectivity strength in the right orbitofrontal gyrus and age of onset in RLS patients. The $\mathrm{x}$-axis is age of onset and the $\mathrm{y}$-axis is connectivity strength in the right orbito-frontal gyrus.

compared to healthy controls(Group mean and std. values across subjects of FC values across all voxels in the cluster were $0.3077 \pm 0.2083$ for RLS and $0.1176 \pm 0.2073$ for controls at $(62,-32,44)$ in MNI coordinate with 1400 cluster size showing Max t value of 3.7687) (Table 2, Fig. 2).

There were two significant correlations when analysis was conducted between the patients' characteristics and the connectivity strength of the right orbitofrontal gyrus. The age of onset was negatively correlated with connectivity strength between the right anterior insula and right orbitofrontal gyrus $(r=-0.361, p=0.049)$ (Fig. 3) and the duration of symptoms was positively correlated with connectivity strength between the right anterior insula and right orbitofrontal gyrus $(\mathrm{r}=0.412, \mathrm{p}=0.024)$ (Fig. 4). Connectivity strength was not correlated with any other characteristics such as K-IRLS total $(r=-0.007, p=0.971)$, or sub-scores of K-IRLS $(r=0.001, p=0.997)$. However, the significant correlations were not maintained when multiple comparisons were considered. 


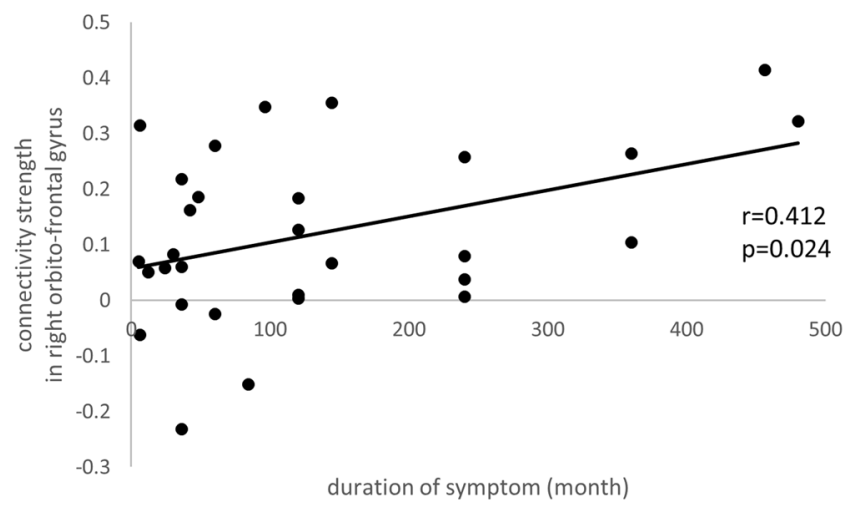

Figure 4. The correlation between the connectivity strength in the right orbitofrontal gyrus and duration of symptoms in RLS patients. The $\mathrm{x}$-axis is the duration of symptoms and the $\mathrm{y}$-axis is connectivity strength in the right orbito-frontal gyrus.

\section{Discussion}

This study showed several changes in SN FC, particularly in the right anterior insula of RLS patients. The research team operated under the hypothesis that the SN plays a determining factor in identifying salient stimuli, which in turn could influence RLS symptoms. One suggestion is that the identification of salient stimuli may be incorrectly processed in RLS patients, resulting in alterations in SN FC. Test results showed increased SN FC in the right postcentral gyrus and bilateral orbitofrontal gyrus, while SN FC in the cerebellum decreased.

Functional connectivity with the salience network. The SN usually functions as a switch between the default mode network and the executive network by detecting salient events and coordinating other brain networks ${ }^{14,33,34}$. According to Putch's review ${ }^{34}$, the SN and the Central Executive Network (CEN) increase in activity when saliency is detected and cognitive tasks which require attention to external stimuli are present ${ }^{35}$, whereas $\mathrm{DMN}$ activity is suppressed ${ }^{36,37}$. The $\mathrm{SN}$ is thought to be responsible for detecting and filtering information necessary to maintain goal-directed behavior by shifting attention between external and internal processing ${ }^{38,39}$. Therefore, the alteration of FC with the SN could lead to the failure of the switching or filtering processes, so the sensory related salience signals which are generated internally would be detected by the SN and improperly processed by other brain networks (e.g. executive function). Therefore, the fact that the brain areas in this study showed aberrant connectivity with the right anterior insula compared to healthy controls could be viewed as a result of incorrect processing of saliency, or as a change in FC with the SN. If the anterior insula is unable to handle this role on its own, it could lead to the recruitment of other brain networks to process the information which might also result in flawed or incomplete processing after being detected by the SN.

The postcentral gyrus is a representative area for somatosensory processing and receives the bulk of the thalamocortical projections from the brain's sensory input fields ${ }^{31}$. So, the enhanced connectivity between the right anterior insula (associated with awareness of viscerosensory information ${ }^{32}$ and salience processing in inhibitory control ${ }^{33}$ ) and the postcentral gyrus could mean that salience stimuli are closely connected to the somatosensory area in individuals with RLS. This area can process sensory information when RLS patients perceive and inhibit sensory stimuli. One possible explanation is that when sensory-related saliency is generated internally, it can be easily detected and transferred to the somatosensory areas, which would then resolve and inhibit said saliency.

The orbitofrontal area is one of the representative areas for the executive function and is usually involved in inhibition ${ }^{33,40}$. It plays a key role in error monitoring and correction. Damage to the orbitofrontal cortex in humans can hinder learning and reverse stimulus-reinforcement associations. In addition, damage to this area has been shown to result in reduced control over emotions, limited awareness of the moral implications of actions, and poor decision making ${ }^{41,42}$. The orbitofrontal cortex is also activated to resolve ambiguity. Internally generated stimuli, which could include the urge to move in RLS patients, are likely to increase ambiguity in processing. Therefore, the activation of the orbitofrontal network would be involved in order to resolve this ambiguity. It also represents secondary salience signals that reflect body sensations by way of the medial orbitofrontal network (or 'visceromotor') which helps control internal body states ${ }^{43}$.

Increased FC with the $\mathrm{SN}$ is regularly observed in the sleep-deprived brain ${ }^{44}$ as well as patients with insomnia who are in hyper-arousal state ${ }^{45}$. Therefore, it is possible that the alterations in SN FC as witnessed by our team may be associated with the hyper-arousal state that can occur as a result of RLS ${ }^{46}$.

Limitations. Although there are valuable findings obtained in this study, there are several limitations as well. First, research was conducted with a small sample size, so a firm conclusion is difficult to reach at this time. Additionally, only the RLS group was compared to the controls even though there may be other patient groups which share similar brain mechanism dysfunctions (e.g. somato-sensory or sleep disorders) as those with RLS. Sleep quality and mood swings in RLS patients may also affect the results. Finally, our study only conducted resting-state functional connectivity analysis using fMRI. The limitations listed above mean that results of this study may be vulnerable to misinterpretation or generalization because of their lack of specificity. Additional 
multi-modal approaches would help to provide clarification and alternate perspectives. Therefore, further research would be necessary to resolve these issues and provide a comprehensive outline of the topic at hand.

\section{Conclusion}

Despite these limitations, the findings of this study suggest that the FC with the SN in RLS patients may be associated with several changes in the overall FC of the brain, which could have an influence on the ability to process the saliency of information. We confirmed that other brain areas were activated together for the additional processing of information. This is particularly relevant when considering sensory information processing and inhibition mechanisms. It could give valuable insight for understanding the pathophysiology of RLS patients.

Received: 23 December 2019; Accepted: 20 April 2020;

Published online: 06 May 2020

\section{References}

1. Allen, R. P. et al. Restless legs syndrome: diagnostic criteria, special considerations, and epidemiology: a report from the restless legs syndrome diagnosis and epidemiology workshop at the National Institutes of Health. Sleep Med 4, 101-119 (2003).

2. Allen, R. P. et al. Restless legs syndrome prevalence and impact: REST general population study. Arch. Intern. Med. 165, 1286 (2005).

3. Cho, Y. et al. Epidemiology of restless legs syndrome in Korean adults. Sleep 31, 219-223 (2008).

4. Winkelmann, J. et al. Treatment of restless legs syndrome: Evidence-based review and implications for clinical practice (Revised 2017)(section sign). Mov. Disord. 33, 1077-1091, https://doi.org/10.1002/mds.27260 (2018).

5. Lee, B. Y. et al. Involvement of the central somatosensory system in restless legs syndrome: A neuroimaging study. Neurology $\mathbf{9 0}$, e1834-e1841, https://doi.org/10.1212/WNL.0000000000005562 (2018).

6. Ku, J. et al. Default mode network disturbances in restless legs syndrome/Willis-Ekbom disease. Sleep Med 23, 6-11, https://doi. org/10.1016/j.sleep.2016.05.007 (2016).

7. Ku, J. et al. Functional connectivity alternation of the thalamus in restless legs syndrome patients during the asymptomatic period: a resting-state connectivity study using functional magnetic resonance imaging. Sleep Med 15, 289-294, https://doi.org/10.1016/j. sleep.2013.09.030 (2014).

8. Ku, J. et al. Diurnal variation of default mode network in patients with restless legs syndrome. Sleep Med 41, 1-8, https://doi. org/10.1016/j.sleep.2017.09.031 (2018).

9. Gorges, M. et al. Patterns of increased intrinsic functional connectivity in patients with restless legs syndrome are associated with attentional control of sensory inputs. Neurosci Lett 617, 264-269, https://doi.org/10.1016/j.neulet.2016.02.043 (2016).

10. Rizzo, V. et al. Impairment of sensory-motor integration in patients affected by RLS. J Neurol 257, 1979-1985, https://doi. org/10.1007/s00415-010-5644-y (2010).

11. Cho, Y. W. et al. Quantitative sensory test for primary restless legs syndrome/Willis-Ekbom disease using the current perception threshold test. Sleep Med 30, 19-23, https://doi.org/10.1016/j.sleep.2016.03.003 (2017).

12. Yang, H. et al. A study for the mechanism of sensory disorder in restless legs syndrome based on magnetoencephalography. Sleep Med 53, 35-44, https://doi.org/10.1016/j.sleep.2018.07.026 (2018).

13. Walter, H. et al. Further evidence for aberrant prefrontal salience coding in schizophrenia. Front Behav Neurosci 3, 62, https://doi. org/10.3389/neuro.08.062.2009 (2010).

14. Menon, V. \& Uddin, L. Q. Saliency, switching, attention and control: a network model of insula function. Brain Struct Funct 214, 655-667, https://doi.org/10.1007/s00429-010-0262-0 (2010).

15. Hening, W. A., Allen, R. P., Washburn, M., Lesage, S. \& Earley, C. J. Validation of the Hopkins telephone diagnostic interview for restless legs syndrome. Sleep Med 9, 283-289, S1389-9457(07)00186-4 [pii] 10.1016/j.sleep.2007.04.021 (2008).

16. Allen, R. P. et al. Restless legs syndrome/Willis-Ekbom disease diagnostic criteria: updated International Restless Legs Syndrome Study Group (IRLSSG) consensus criteria-history, rationale, description, and significance. Sleep Med 15, 860-873, https://doi. org/10.1016/j.sleep.2014.03.025 (2014).

17. Yang, J. et al. The Reliability and Validity of the Korean Versions of the International Restless Legs Scale and the Restless Legs Syndrome Quality of Life Questionnaire. J Korean Neurol Assoc 28, 263-269 (2010).

18. Walters, A. S. et al. Validation of the International Restless Legs Syndrome Study Group rating scale for restless legs syndrome. Sleep Med 4, 121-132, S1389945702002587 [pii] (2003).

19. Cho, Y. W., Song, M. L. \& Morinc, C. M. Validation of a korean version of the insomnia severity index. J Clin Neurol 10, 210-215, https://doi.org/10.3988/jcn.2014.10.3.210 (2014).

20. Sohn, S. I., Kim, D. H., Lee, M. Y. \& Cho, Y. W. The reliability and validity of the Korean version of the Pittsburgh Sleep Quality Index. Sleep Breath 16, 803-812, https://doi.org/10.1007/s11325-011-0579-9 (2012).

21. Cho, Y. W. et al. The reliability and validity of the Korean version of the Epworth sleepiness scale. Sleep Breath 15, 377-384, https:// doi.org/10.1007/s11325-010-0343-6 (2011).

22. Cox, R. W. AFNI: software for analysis and visualization of functional magnetic resonance neuroimages. Comput Biomed Res 29, 162-173 (1996).

23. Palaniyappan, L. \& Liddle, P. F. Does the salience network play a cardinal role in psychosis? An emerging hypothesis of insular dysfunction. J Psychiatry Neurosci 37, 17-27, https://doi.org/10.1503/jpn.100176 (2012).

24. Pelletier-Baldelli A, Bernard JA, Mittal VA. Intrinsic Functional Connectivity in Salience and Default Mode Networks and Aberrant Social Processes in Youth at Ultra-High Risk for Psychosis. PLoS One. 2015;10(8):e0134936. Epub 2015/08/08.

25. Fan J, MXet al. Resting-state functional connectivity between right anterior insula and right orbital frontal cortex correlate with insight level in obsessive-compulsive disorder. Neuroimage Clin. 2017;15:1-7. Epub 2017/05/02.

26. Sato, W. et al. Resting-state neural activity and connectivity associated with subjective happiness. Sci Rep 9, 12098, https://doi. org/10.1038/s41598-019-48510-9 (2019).

27. Friston, K. J., Williams, S., Howard, R., Frackowiak, R. S. \& Turner, R. Movement-related effects in fMRI time-series. Magn Reson Med 35, 346-355, https://doi.org/10.1002/mrm.1910350312 (1996).

28. Satterthwaite, T. D. et al. An improved framework for confound regression and filtering for control of motion artifact in the preprocessing of resting-state functional connectivity data. Neuroimage 64, 240-256, https://doi.org/10.1016/j. neuroimage.2012.08.052 (2013).

29. Power, J. D., Barnes, K. A., Snyder, A. Z., Schlaggar, B. L. \& Petersen, S. E. Spurious but systematic correlations in functional connectivity MRI networks arise from subject motion. Neuroimage 59, 2142-2154, https://doi.org/10.1016/j.neuroimage.2011.10.018 (2012).

30. Allen, R. P., Kushida, C. A. \& Atkinson, M. J. Factor analysis of the International Restless Legs Syndrome Study Group's scale for restless legs severity. Sleep Med 4, 133-135 (2003).

31. Padberg, J. et al. Thalamocortical connections of parietal somatosensory cortical fields in macaque monkeys are highly divergent and convergent. Cereb Cortex 19, 2038-2064, https://doi.org/10.1093/cercor/bhn229 (2009). 
32. Kotani, Y. et al. The role of the right anterior insular cortex in the right hemisphere preponderance of stimulus-preceding negativity (SPN): an fMRI study. Neurosci Lett 450, 75-79, https://doi.org/10.1016/j.neulet.2008.11.032 (2009).

33. Ghahremani, A., Rastogi, A. \& Lam, S. The role of right anterior insula and salience processing in inhibitory control. J Neurosci 35, 3291-3292, https://doi.org/10.1523/JNEUROSCI.5239-14.2015 (2015).

34. Putcha, D., Ross, R. S., Cronin-Golomb, A., Janes, A. C. \& Stern, C. E. Salience and Default Mode Network Coupling Predicts Cognition in Aging and Parkinson's Disease. J Int Neuropsychol Soc 22, 205-215, https://doi.org/10.1017/S1355617715000892 (2016).

35. Dosenbach, N. U. et al. A core system for the implementation of task sets. Neuron 50, 799-812, https://doi.org/10.1016/j. neuron.2006.04.031 (2006).

36. Greicius, M. D., Krasnow, B., Reiss, A. L. \& Menon, V. Functional connectivity in the resting brain: a network analysis of the default mode hypothesis. Proc Natl Acad Sci USA 100, 253-258, https://doi.org/10.1073/pnas.0135058100 (2003).

37. Raichle, M. E. et al. A default mode of brain function. Proc Natl Acad Sci USA 98, 676-682, https://doi.org/10.1073/pnas.98.2.676 (2001).

38. Menon, V. Large-scale brain networks and psychopathology: a unifying triple network model. Trends Cogn Sci 15, 483-506, https:// doi.org/10.1016/j.tics.2011.08.003 (2011).

39. Seeley, W. W. et al. Dissociable intrinsic connectivity networks for salience processing and executive control. J Neurosci 27, 2349-2356, https://doi.org/10.1523/JNEUROSCI.5587-06.2007 (2007).

40. Dosenbach, N. U., Fair, D. A., Cohen, A. L., Schlaggar, B. L. \& Petersen, S. E. A dual-networks architecture of top-down control. Trends Cogn Sci 12, 99-105, https://doi.org/10.1016/j.tics.2008.01.001 (2008).

41. Anderson, S. W., Bechara, A., Damasio, H., Tranel, D. \& Damasio, A. R. Impairment of social and moral behavior related to early damage in human prefrontal cortex. Nat Neurosci 2, 1032-1037, https://doi.org/10.1038/14833 (1999).

42. Grafman, J. et al. Frontal lobe injuries, violence, and aggression: a report of the Vietnam Head Injury Study. Neurology 46, 1231-1238, https://doi.org/10.1212/wnl.46.5.1231 (1996).

43. Barrett, L. F. \& Bar, M. See it with feeling: affective predictions during object perception. Philos Trans R Soc Lond B Biol Sci 364, 1325-1334, https://doi.org/10.1098/rstb.2008.0312 (2009).

44. Fang, Z. et al. Altered salience network connectivity predicts macronutrient intake after sleep deprivation. Sci Rep 5, 8215, https:// doi.org/10.1038/srep08215 (2015)

45. Liu, C. H. et al. Increased Salience Network Activity in Patients With Insomnia Complaints in Major Depressive Disorder. Front Psychiatry 9, 93, https://doi.org/10.3389/fpsyt.2018.00093 (2018).

46. Lanza, G. \& Ferri, R. The neurophysiology of hyperarousal in restless legs syndrome: Hints for a role of glutamate/GABA. Adv Pharmacol 84, 101-119, https://doi.org/10.1016/bs.apha.2018.12.002 (2019).

\section{Acknowledgements}

"This research was supported by Basic Science Research Program through the National Research Foundation of Korea (NRF) funded by the Ministry of Education (2017R1D1A3B03031021)".

\section{Author contributions}

Jeonghun Ku, Keun Tae Kim and Yong Won Cho designed the research. Yeong Seon Lee, and HyukWon Chang performed the experiments and collected the data. Jeonghun $\mathrm{Ku}$ and Yeong Seon Lee analysed the data. Jeonghun $\mathrm{Ku}$, Keun Tae Kim and Yong Won Cho wrote and edited the paper. All authors reviewed manuscript and approved the final version of the manuscript.

\section{Competing interests}

The authors declare no competing interests.

\section{Additional information}

Supplementary information is available for this paper at https://doi.org/10.1038/s41598-020-64641-w.

Correspondence and requests for materials should be addressed to Y.W.C.

Reprints and permissions information is available at www.nature.com/reprints.

Publisher's note Springer Nature remains neutral with regard to jurisdictional claims in published maps and institutional affiliations.

(c) (i) Open Access This article is licensed under a Creative Commons Attribution 4.0 International

License, which permits use, sharing, adaptation, distribution and reproduction in any medium or format, as long as you give appropriate credit to the original author(s) and the source, provide a link to the Creative Commons license, and indicate if changes were made. The images or other third party material in this article are included in the article's Creative Commons license, unless indicated otherwise in a credit line to the material. If material is not included in the article's Creative Commons license and your intended use is not permitted by statutory regulation or exceeds the permitted use, you will need to obtain permission directly from the copyright holder. To view a copy of this license, visit http://creativecommons.org/licenses/by/4.0/.

(C) The Author(s) 2020 